\title{
Aristotelian Essentialism: Essence in the Age of Evolution Christopher J. Austin
}

\begin{abstract}
The advent of contemporary evolutionary theory ushered in the eventual decline of Aristotelian Essentialism (Æ) - for it is widely assumed that essence does not, and cannot have any proper place in the age of evolution. This paper argues that this assumption is a mistake: if $Æ$ can be suitably evolved, it need not face extinction. In it, I claim that if that theory's fundamental ontology consists of dispositional properties, and if its characteristic metaphysical machinery is interpreted within the framework of contemporary evolutionary developmental biology, an evolved essentialism is available. The reformulated theory of $\mathbb{E}$ offered in this paper not only fails to fall prey to the typical collection of criticisms, but is also independently both theoretically and empirically plausible. The paper contends that, properly understood, essence belongs in the age of evolution.
\end{abstract}

Within contemporary philosophy of biology, there is perhaps no greater maligned theory than Aristotelian Essentialism (ÆE). Now that the rosy dawn of Aristotelian metaphysics has faded into twilight ${ }^{1}$, citing the essence of an organism as an explanatory principle is indicative either of a rather hopeless scientific naiveté

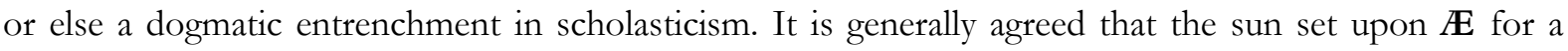
simple, yet powerful reason: the advent of evolutionary theory. According to the implications of that theory, kind-essences are an ontological superfluity which the world not only has no need of, but simply cannot countenance. However, evolutionary theory has recently had its own paradigm shift, ushered in with the rise of the union between it and developmental theory. With its increasing emphasis on modular, structural explanations of morphological novelty and variation, evolutionary developmental biology (evodevo) has arguably prompted a substantial reshaping of our understanding of the very nature of biological individuals. In light of this reformation, the question naturally arises: what is it to be the what-it-is-to-be of an organism? In what follows, I suggest that the answer to that question is one best interpreted within the ontological framework of $Æ$. I contend that, properly understood, essence belongs in the age of evolution.

\section{Aristotelian Essentialism vs. Evolution}

First things first: what exactly is $\mathbb{E}$ ? One can find many distinct (though often overlapping) definitions in the literature, but here, for the sake of simplicity, and without wishing to rehearse decades of debate, I focus on a simple three-point definition. An Aristotelian essence is (a) comprised of a natural set of intrinsic properties which (b) constitute generative mechanisms for particularised morphological development which (c) are shared among groups of organisms, delineating them as members of the same 'kind'. Regarding (a), the set of properties that comprise an essence and define a natural kind cannot be extrinsic, or relational properties - abstract properties of phylogenetic lineage or interbreeding relations, etc. - and their being a collection must not be a result of our conceptual practices (on account of discipline convention or theoretical interests), but instead reflect a structural grouping that is mind-independent: the set of properties that define a natural kind are chosen by nature, not nous. Regarding (b), the properties that comprise an essence are causal properties, teleologically "directed toward" particular anatomical and eidonomical ends.

${ }^{1}$ I borrow this colourful phrasing from Hacking (2007). 
Thus $~ Æ$ presents, as Lennox (1987: 340) puts it, a type of 'teleological essentialism': these groups of properties are causally responsible for the continual shaping and organising of the particularised ontogenic development of the organisms which possess them. ${ }^{2}$ Regarding (c), being possessed by a great number of organisms and reflecting the ways in which nature is "carved at the joints", these property groupings sort organisms into kinds, functioning as inductively rich 'information stores' about their members' typical morphological development. ${ }^{3}$ That said, although the essences of $\mathbb{E}$ are certainly typological - in that they sort organisms into developmental types - they are not taxonomical: strictly speaking, $\mathbb{E}$ is a thesis about the source and nature of ontogenic development, not a methodological prescription for classificatory

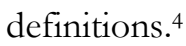

Importantly, note that in endorsing (a)-(c), $\mathbb{E}$ entails a rather particular ontological commitment concerning fundamentality. For the defender of $\mathbb{E}$, what is fundamental about the world (and is thus primitively explanatory with respect to the biological realm) is stability, or invariance - it is the unchanging, shared set of kind-defining intrinsic properties which ontologically "lie at the bottom" of organisms. Variation on the other hand, according to $Æ$, is therefore non-fundamental: the wide range of property mosaics peppered throughout the organisms that populate the natural world are an ontological result of, and hence must be explained by reference to, the invariant natures - or essences - of those organisms. It is the endorsement of this ontological priority, and its declaration of the dominance of the unchanging over the changing, which has engendered what has long been viewed as the most singular error of any essentialist theory in biology: for $\mathbb{E}$ 's commitment to the so-called 'natural state model' is nearly universally understood as standing in direct conflict to the contemporary cornerstone of our understanding of the biological realm - namely, the theory of evolution and the process of natural selection. Indeed, $\mathbb{E}$ has been thought by many to be "...precisely the 'typological' perspective... that Darwin had to displace". 5

Because I take it that $\mathbb{E}$ 's commitment to the natural state model is the primary source of the contemporary distaste for that theory, I will consider it in detail here. When the 'natural state model' is mentioned, it is likely that most will have in mind Mayr's (1976: 27) popular (and overtly Platonic) phrasing that, on this model, “... [t] here are a limited number of fixed, unchangeable 'ideas' underlying the observed variability [in nature], with the eidos (idea) being the only thing that is fixed and real, while the observed variability has no more reality than the shadows of an object on a cave wall"; here the essences of natural kinds are the Platonic eidoi, while the various distinct instances of the morphogenetic profiles of those kinds are the observed variability. This statement is rather incendiary but, as gestured at in the outline above, the general idea is correct, and $\mathbb{E}$ is committed to it: differentiation of specific property exemplification among members of the same natural kind comes about via various "accidental" exploitations of its essential properties, these properties themselves being both (kind-) defining and unchanging. But of course, by the lights of evolutionary theory, this view of the world simply has it backwards: it is variation which is primitive, not fixity. As Gould (1985:160-161) puts it, "[v]ariation is the raw material of evolutionary change [which] represents the fundamental reality of nature, not an accident about a created norm". ${ }^{6}$

This view is bolstered by the fact that, just as one would expect if the stasis that the natural state model posits were not fundamental, the vast majority of empirical evidence suggests that there are no ontologically privileged sets of properties - genetic or otherwise - which all members of (purported)

\footnotetext{
${ }^{2}$ Cf. Wilkins (2013), Devitt (2008).

3 As Elder (2008: 345) notes: "If a plurality of organisms is to populate a genuine natural kind...more is needed than just that the same phenotypic traits crop up in member after member of the plurality. The same traits must recur across all the organisms for a common reason".

${ }^{4}$ Cf. Lennox (2001), Pellegrin (1987), and Balme (1987).

${ }^{5}$ Griffiths (2002: 77).

${ }^{6}$ Cf. Okasha (2002: 191).
} 
natural kinds share. ${ }^{7}$ The lack of such evidence, as Okasha (2002: 196) rightly points out, has led "virtually all philosophers of biology [to] agree that...it is simply not true that the groups of organisms that working biologists treat as [members of the same natural kind] share a set of common morphological, physiological or genetic traits which set them off from other [kinds]". 8 And what's perhaps worse, not only is it nearly universally agreed upon that the invariability posited by the natural state model is nowhere to be found, it is likewise agreed that such invariability runs counter to the very core of the evolutionary world-view: for the sine quo non of the process of natural selection is the existence of a substantial amount of phenotypic and genetic heterogeneity among (purported) members of the same natural kind. ${ }^{9}$

Furthermore, as Sober (1980: 374-377) convincingly argues, even if there were a stable and unchanging set of properties shared among members of a delineated natural kind, those properties would be incapable of playing the role that $\mathbb{E}$ requires of them. According to (b), the essence of a natural kind is an intrinsic set of causal, goal-directed properties which define a particular developmental path towards a specific 'morphological profile' (according to its kind). In the parlance of the natural state model, the essence of a natural kind is causally productive of an intrinsically privileged developmental plan, one which represents the 'natural state' of the members of that kind, with variations on this natural state representing destructive deviations attributable to the pervading un-natural causal influence of an organism's environment. However, while the discovery of the phenomenon of phenotypic plasticity has taught us that a developing organism's environment is an important and major source of phenotypic variation, it has also taught us that there simply is no such thing as an environment-independent phenotypic trait - and hence, by extension, no such thing as a natural state, produced purely by an intrinsically specified pathway of particular morphological development. ${ }^{10}$ Indeed, more and more empirical research suggests that the developmental specification of morphological features via environmental stimuli is not only a functionally ubiquitous phenomenon, but one that may play a vitally important part in the evolutionary process. ${ }^{11}$ Thus, even if we were to grant that there exist shared sets of intrinsic properties among groups of organisms that demarcated them as members of a particular natural kind, $\mathbb{E}$ 's requirement that this intrinsic essence must function - and do so in some way independently of the extrinsic environment - as the "prime mover" with respect to an organism's specified morphological development is a theoretical demand which the biological realm does not and cannot meet.

\section{The Evolution of Essentialism}

These critiques of the natural state model collectively function as a powerful reason for abandoning the metaphysical machinery of $\mathbb{E}$ - this I do not wish to deny. What I deny, as I will argue below, is that these critiques must sound the theory's death knell. To my mind, the lesson they teach is not that $\mathbb{E}$ ought to be extinct, but rather that if the theory is to survive in a contemporary landscape it, like all else, must evolve. Thus I've no hesitancy in affirming that the objections of the previous section are devastating for a certain naïve, primitive form of $\mathbb{E}$ - perhaps even for the form which Aristotle himself advanced. Indeed, I am more than happy to let the specific letter of that primitive progenitor pass away, if there is available a novel, more sophisticated contemporary form of the theory which properly retains the former's spirit. It is my contention that just such a theoretical advancement is available, once we understand that the Aristotelian dunamis which lies at the heart of that ancient form of essentialism is but the obscure, imprecise ancestor of the subsequently developed, specified and complex contemporary

\footnotetext{
7 This lack of evidence has arguably led to the formulation of the 'Homeostatic Property Cluster' view of natural kinds - see Boyd (1999), Wilson (1999), and Wilson, Barker, and Brigandt (2007).

8 See also Griffiths (2002), Hull (1992), and Sober (1980).

${ }^{9}$ Cf. Wilson (1999: 190), and Okasha (2002: 197).

${ }^{10}$ For a general overview, see Whitman \& Agrawal (2009) and Schlichting \& Smith (2002).

11 See Fusco \& Minelli (2010) and West-Eberhard (2003).
} 
concept of dispositionality. The evolution of this central concept, I suggest, capably courts the viable evolution of $\mathbb{E}$.

Dispositional properties are inherently causal properties - they are responsible for the coming about of particular states of affairs ('manifestation states') upon the occurrence of some other state of affairs ('stimulus conditions'). ${ }^{12}$ These properties function as ontological "switches" of sorts, causally mediating the influence of certain activating conditions to produce particular states of affairs. The property of 'negative charge', for instance, is widely understood dispositionally - when its bearers meet with a like-charged particle (its stimulus condition), they repel with a particular momentum (its manifestation state). Of course, this (and examples of its ilk from "fundamental" ontologies) are the exception, for while it's possible for dispositions to be realised by a single material element, they are more often than not realised by an entire system, or complex network of interacting elements: if upon receiving the appropriate conditions that network initiates a sustained step-wise progressive interplay among its nodes which leads to their production of a particular end state, that complex of elements realises a dispositional property. ${ }^{13}$

Thus dispositions are functionally defined with respect to their specific stimulus/manifestation pairs: whatever performs the function of causally mediating the occurrence of a specific manifestation state upon the occurrence of specific stimulus conditions is an instance of the disposition defined by that particular pair. ${ }^{14}$ Importantly, when we designate a particular structure as an instance of a functionally defined property, we are operating at a certain level of abstraction - one that eschews the more specific details of the causal pathway by which that function is performed and focuses on the general end states between which that pathway runs. When a dispositional property is realised by a particular system then, the pathway from 'stimulus' to 'manifestation' often "reaches over" a wide, multi-stage causal gap - thus, when such a gap is reliably and repeatedly bridged (upon the appropriate conditions being realised), we are afforded evidence of the existence of these properties. ${ }^{15}$

In abstracting to these end states, we not only abstract away from the particulars of that pathway that is, the various links comprising the causal chain between those states - but also the various particular ways in which that pathway might be traversed. Accordingly, because there are many distinct instances of a particular type of stimulus condition which might lead to distinct instances of a particular type of manifestation state, the two states which define a disposition are determinables, not determinates. When a dispositional property is realised by a system then, that system is capable of producing a wide, gradientlike range of quantitatively distinct manifestation states, each representing a particular instance of its manifestation type, according to its particular stimulus input. Dispositional properties are therefore functional in a second sense, in that they establish a functional relation between a set of input values that is, particular determinate instances of certain determinable stimulus conditions - and a set of output

${ }^{12}$ Dispositions are often contrasted with 'categorical' properties - those whose nature must be imbued with causality from higher-order laws of nature, or else some flotilla of possible worlds. For discussions of the distinction, see Ellis (2010), Oderberg (2009), and Cross (2005).

${ }^{13}$ Even the seemingly simple philosopher's paragon of dispositionality - 'fragility' - is realised (in most cases) by a complex physical microstructure, and 'breaking' is in fact a complex, multi-stage process featuring the aligning of various micro-events that represent decreasing degrees of structural integrity.

14 That dispositional properties are responsible for establishing this type of causal connection between two states is the basis for their ubiquitous assignment as truthmakers for subjunctive conditionals (especially counterfactuals). However, spelling out precisely what the truthmaking role is, and showing that dispositional properties play it with respect to those conditionals turns out to be exceptionally tricky. As it happens, I won't be making use of that concept here, as I've no need for it. For a good discussion of the related issues, see Austin (2015b) and Eagle (2009). 15 I've said "reliably and repeatedly" purposely here, as dispositional properties do not necessitate their manifestations - a fact ensured by the possibility of so-called 'masks', properties or processes which interrupt the causal activity of dispositional properties. See Eagle (2009), Schrenk (2010), and Mumford \& Anjum (2011) for good discussions of the issue. 
values - that is, particular determinate instances of certain determinable manifestation states. ${ }^{16}$ In other words, more specifically, dispositional properties establish a causal link of functional co-variance of state-values between two variables. ${ }^{17}$

Because dispositions reliably and repeatedly produce particular end states, they are often understood as teleological, goal-directed properties - they are causally "directed toward", and are thus for those ends. When a system is goal-directed toward a particular end, it exhibits the phenomenon of persistence $e^{18}$ : it maintains the production of its end state "as a result of changes occurring in the system that compensate for any disturbances taking place (provided these are not too great) either within or [external] to the system, disturbances which, were there no compensating changes elsewhere, would prevent the realisation of the [end state]". ${ }^{19}$ The causal process which dispositional properties initiate and mediate is characterised by this sort of "course correcting" towards a particular end state, which they do in a systematic and non-accidental fashion - that is to say, reliably and repeatedly, over a wide-range of changes/perturbations. ${ }^{20}$

In the framework of $\mathbb{E}$, organisms are ontologically sorted into natural kinds in virtue of sharing sets of causal properties which both generate and subsequently shape their morphological development it is my contention that these "powerful" properties are dispositional properties. The relevant question then is: is $\mathbb{E}$ 's ontology, armed with this contemporary gloss, consistent with our current understanding of the biological realm? I propose that it $i s$ - for my claim is that the advent of evolutionary developmental biology has afforded us a unique view of that realm, one whose requisite ontology is dispositional, and whose foundational principles just are those of $\mathbb{E}$ : for evo-devo is a framework in which morphological variation is derived from invariant, functional causal mechanisms which serve as highly conserved "deep homologies", underwriting a vast array of organismal diversity. In order to make the argument for the viable evolution of $\mathbb{E}$ by way of evo-devo, I turn now to the specifics.

Recall that, if $\mathbb{E}$ is to be plausible, there must be a set of causal capacities - on my gloss, a set of dispositional properties - jointly responsible for an organism's particularised morphological development. The question is: in any particular organism, are there discrete properties which function as generative mechanisms with respect to particular phenotypic traits? According to evo-devo, there most certainly $\operatorname{are}^{21}$ : for one of the guiding principles of that framework is the modularity of development, according to which "...developmental systems are decomposable into components that operate according to their own intrinsically determined principles". 22 These separable and distinct 'developmental modules' are identified with highly internally integrated genetic regulatory networks which "interpret" particular intra- and intercellular signalling into downstream (spatial and temporal) regulatory control via the production of transcription factors, resulting in patterns of expression which specify the particularised developmental pathways of discrete morphological structures. ${ }^{23}$ In other words, these modules are each responsible for the specified development of a particular morphological structure in a developing organism, and

\footnotetext{
${ }^{16}$ In the dispositions literature, this fact is often referred to as dispositions being “multi-track". See Martin (2008), Manley and Wasserman (2008), Jacobs (2011), and Vetter (2013) for fuller discussions.

${ }^{17}$ This is a species of the relation that Lewis (2000: 190) called 'causal influence' which forms the conceptual bedrock of Woodward's $(2003 ; 2010)$ influential theory of causation.

18 Of course, not all dispositions are strongly goal-directed in this sense, but the ones which will concern us here namely, those that populate the biological realm - certainly are.

${ }^{19}$ Nagel (1977: 272).

${ }^{20}$ See Walsh (2012), and Mayr (1992).

${ }^{21}$ Indeed, as many have now argued, 'modularity' may very well be a necessary requisite for the process of evolution: we may need variability to occur within discrete elements which doesn't affect other elements if organisms are going to survive mutations and be subsequently subject to selection pressures. See Lewontin (1978) and Altenberg (1995).

22 Müller (2008: 10).

${ }^{23}$ See Winther (2001), Bolker (2000), and Von Dassow \& Munro (1999).
} 
accordingly, are individuated with respect to the structure whose development they are causally responsible for, evidence for which is gathered either by ectopic expression experiments ${ }^{24}$, or else by the principled decomposition of genotype-phenotype mappings. ${ }^{25}$ Indeed, although discovering the niceties of the regulatory architecture which comprises these developmental modules is an interesting and thus far fruitful research programme (especially with respect to its prowess in establishing molecular-based phylogenetic lineages), what is most important is their generative specificity with respect to particular morphological structures. This is underscored by the fact that these modules' role in the production of such structures is characterised by a highly robust, degenerative process - one underwritten by their constitutive genetic regulatory networks' ability to maintain integrity by means of its non-isomorphic elements becoming isofunctional ${ }^{26}-$, and is one which, over time, and in successive generations, may become autonomised, gaining a kind of independence from their (original) underlying genetic mechanisms. ${ }^{27}$ Accordingly, causal explanations of the development of the structures associated with these modules eventually operate at high 'causality horizons' - that is, at explanatory levels "above" the workings of its molecular constituents. ${ }^{28}$

Recall that if $\mathbb{E}$ is correct, the ontological ground floor of organisms consists in collections of properties which are (jointly) causally responsible for their specified morphological development - that is, in collections of dispositional properties, each "directed toward" the development of a particular morphological structure. Even on the general reading just given, I think it's easy to see that $\mathbb{E}$ is consistent with evo-devo: the fundamental ontological postulates of the latter -'developmental modules' - can be conceptualised as instances of the ontological cornerstones of the former. In other words, from the Aristotelian point of view, developmental modules are dispositional properties. ${ }^{29}$ The developmental modules of evo-devo are causally responsible for the specified production of their associated morphological structures in developing organisms in virtue of their serving as a functional bridge between intra- and inter-cellular signalling and specific downstream genetic expression patterns which initiate particularised developmental pathways resulting in the formation of those structures. These modules therefore function as ontological "switches", causally mediating the influence of certain activating conditions to produce particular states of affairs: given the appropriate stimulus conditions, developmental modules reliably and repeatedly produce particular end states.

And it is with respect to these end states (read: morphological structures) that these modules are functionally individuated: for what is important, theoretically, to the definition of a particular module is not the particularities of the genetic regulatory networks (or hierarchical sets of such networks ${ }^{30}$ ) which undergird its activity, but rather the role it plays in morphological development. Thus, these "higher order" modules are defined after the fashion of dispositional properties, at a certain level of abstraction away from the various complexities of the aforementioned particularities - and are therefore able to be conceptually (and in some cases, as mentioned above, physiologically) "disassociated" from any specific underlying mechanism and constitutive processes. Not only are these modules functionally individuated

\footnotetext{
${ }^{24}$ This technique was especially prominent in Halder et al. (1995); For a general contemporary review in a particular case, see Ashery-Padan \& Gruss (2001). And for an analysis of the expression patterns of modules in particular, see Raff \& Sly (2000).

25 See Wagner \& Altenberg (1996).

${ }^{26}$ See Edelman \& Gally (2001), Von Dassow et al. (2000), and Whitacre \& Bender (2010).

${ }^{27}$ This is discussed with particular examples in Müller \& Newman (1999), and Müller (2003).

${ }^{28}$ See Salazar-Ciudad \& Jernvall (2013) for the concept of 'causality horizons' in explanations of developmental morphology.

${ }^{29}$ Though this general application has been made - in Wagner (2000) and Eble (2005) - , it has only been very briefly stated, and not explored in any depth.

${ }^{30}$ It's plausible that there are at least four distinct "levels" of morphological organisation - see Rasskin-Gutman (2003).
} 
with respect to their end states, but they are plausibly "directed toward" those states - for the degenerative robustness of their underlying networks is an instance of the dispositional, teleological phenomenon of persistence. ${ }^{31}$

Having satisfied the basic conceptual criteria, let us call these developmental modules, in line with the Aristotelian metaphysic, phenomodulatory dispositions. Of course, according to $\mathbb{E}$, it's not enough that there exist collections of causal properties which are responsible for the morphological development of the organisms which possess them - these collections must also be (a) shared among certain sets of organisms, representing a kind of ontological stability, or invariance, and yet somehow function to (b) ontologically ground the "accidental" morphological variation among those sets. The question is: are there shared sets of phenomodulatory dispositions among groups of organisms which function to ontologically underwrite a wide range of their morphological variation? According to evo-devo, there most certainly are: for one of the fundamental posits of that framework is the existence of conserved developmental resources whose inherent plasticity is the causal ground of phenotypic variation. Indeed, in a notable shift from the neo-Darwinian perspective, evo-devo favours a 'structuralist' approach ${ }^{32}$, wherein the diversity of organismal development is understood to be underwritten by a drastically less diverse set of developmental modules which themselves constrain and specify the variability of their associated morphological structures according to their own "generative rules". 33

We now know that the morphological structure produced by a single developmental module, being underwritten by a particular genetic regulatory network, is capable of a wide variety of intra- and inter-cellular environmentally induced phenotypic variation: alterations in 'positional information' consisting mainly of heterochronical and heteropical changes in hormonal and endocrinal signalling results in qualitative alterations of the phenotypic character of that structure; this is the phenomenon of phenotypic plasticity, attested to by the reality (read: quantifiability) of reaction norms. ${ }^{34}$ This is possible because, as evidenced by the evo-devo paragon of HOX regulatory networks, these modules are situated in the "bottleneck" of the process of development, between a host of upstream signalling pathways and a certain set of downstream 'target genes', the latter of which are responsible for producing the "building blocks" of particular morphological structures. ${ }^{35}$ Because these regulatory networks are thus positioned, they function as causal mediators, interpreting cascades of upstream "inputs" into downstream "outputs" via their production of transcription factors which enact (spatial and temporal) regulatory control at the cis-regulatory sites of downstream target genes which directly specify cell fate. ${ }^{36}$ Thus, alterations in their input values are causally correlated with corresponding alterations in their output values - that is, with alterations in the qualitative character of their corresponding morphological structure. ${ }^{37}$

This fact brings with it two important points, the first being that a common set of modules possessed by the members of a single species is able to ground a wealth of their morphological variation for one and the same developmental module can be responsible for a wide variety of phenotypic variation in a particular structure as a result of (broadly construed) environmental influences: as described above, alterations in upstream signalling are interpreted by these modules into downstream regulatory control

\footnotetext{
31 Thus, in the context of dynamical systems theory, the morphological structures associated with these modules are often characterised as 'attractor-states' which shape the "valleys" of an organisms' epigenetic landscape, resulting in many distinct developmental pathways leading to the same end-state. See Jaeger \& Monk (2014), and Striedter (1998).

${ }^{32}$ See Amundson (2005) for an excellent in-depth discussion of the 'structuralist' paradigm and its relation to that of the Modern Synthesis.

${ }^{33}$ Cf. Müller (2008).

34 See West-Eberhard (2003), and Pigliucci (2001).

${ }^{35}$ For more on the concept of the 'developmental hourglass', see Galis \& Metz (2001) and Kalinka et al. (2010)

36 See Mann \& Carroll (2002), Gurdon \& Bourillot (2001), and Tabata (2001).

${ }^{37}$ See Schlichting \& Pigliucci (2002), and Aubin-Horth \& Renn (2009).
} 
over a (shared) set of "building block" target genes, resulting in morphological changes in the development of that structure. Thus on this small, intra-species scale, the morphological variation of particular traits can be accounted for by the causal activity of a shared set of developmentally plastic modules. More important perhaps is the second, rather surprising point that even inter-species morphological variation of particular traits is often grounded in the causal activities of a shared set of developmental modules; this is the so-called phenomenon of deep homology. ${ }^{38}$ Here, as a result of distinct species possessing distinct sets of downstream target genes - and hence, distinct sets of regulatory regions - a single, shared developmental module, producing a particular set of transcription factors, is capable of controlling the specified formation of two seemingly distinct morphological structures. In other words, on evo-devo's 'structuralist' framework, even the more extreme qualitative variation of a particular morphological structure present in two otherwise distinct groups of organisms is often causally underwritten by a shared developmental module.

Importantly, these modules which function as "units of stability" (Eble 2005: 223) underlying the morphological variation among seemingly intractably qualitatively diverse structures in distinct populations are themselves primarily responsible for that variation. On account of their aforementioned roles as causal mediators between upstream signalling pathways and downstream target genes, these shared developmental modules are not only responsible for the development of a particular morphological structure in distinct sets of organisms ${ }^{39}$, but also for variations on that structure: their generative competence in defining a trait's 'morphospace' - the representation of its possible structural permutations - is an intrinsic affair. ${ }^{40}$ In other words, it is their inherent plasticity that grounds these modules' ability to function as the causal basis for the morphological variation on their associated structures, as the specification of their reaction norms - representing the functional relation between upstream signalling and downstream targets - is a role which is "immanent to the system". ${ }^{41}$

On the evo-devo framework then, there exist shared, discrete developmental elements within and among populations of species which are intrinsically causally responsible for the specified development of a certain generalised morphological structure, and which causally control the production of the various particularised forms of that structure. As I hope by now is clear, the operative ontology of that

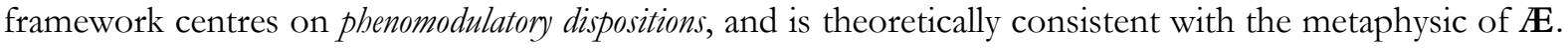
As discussed above, phenomodulatory dispositions are functionally individuated, causally active elements "directed toward" the development of a particular morphological structure. Importantly however, dispositional properties are functional in a second sense, for the causal role they perform is one of mediating and specifying the causal co-variance of determinate state-values between two determinable variables. Thus, the developmental role of morphological modules in functionally mediating between upstream positional signals and the production of a particular morphological structure is a dispositional role - they function to "interpret" specific, determinate collections of a generalised class of stimulus factors into specific, determinate forms of a generalised manifestation state. Due to this inherent plasticity of dispositional properties, one and the same disposition is able to causally underwrite a wide (though restricted) range of end state variations, and hence serve as the shared, "hidden" foundation of a diversity of qualitative attributes among seemingly fundamentally diverse sets of objects. As we have seen,

\footnotetext{
38 See Shubin, Tabin, and Carroll (2009), and Wagner (2007).

${ }^{39}$ Wagner (2014: 92-93) argues that 'character-identity' determination (a) cannot be specified by positional information, given that they are variable in and among instances, and that it likewise (b) cannot be specified by downstream target genes, given not only their similar variability, but also their regulatory dependence upon upstream modules.

${ }^{40}$ Cf. Newman and Müller (2006), and Newman et al. (2006). For the concept of 'morphospace' generally, see Rasskin-Gutman (2005), and McGhee (2006).

${ }^{41}$ Müller (2008: 19).
} 
developmental modules, as the ground of "deep homology", serve this same function, and in the same way: they represent a kind of ontological stability, or invariance which grounds the possibility of subsequent derivative, or "accidental" (in the Aristotelian sense) organismal variation.

\section{Essentialism Evolved}

If, as I have claimed, the evidence of evo-devo points us to the existence of phenomodulatory dispositions, and its general explanatory framework mirrors the foundational metaphysic of $\mathbb{E}$, what then does an evolved essentialism look like? The first, rather general answer, in line with the initial criteria offered, is that an Aristotelian essence is (a) comprised of a set of phenomodulatory dispositions which (b) function as generative modules for particularised morphological development which (c) are shared among groups of organisms, delineating them as members of the same 'kind'. Thankfully, with the above discussion in mind, we can be more specific about these 'essential' properties, both quantitatively and qualitatively. First, quantitatively: an essence of a natural kind must be comprised of a nested, scalar set of a number of phenomodulatory dispositions - ones whose manifestations comprise a wide-ranged set of more "basic" morphological features (such as the general spatio-temporal axis of an organism and its general morphological shape-differentiation) within which lies a more narrow-ranged set whose manifestations comprise more specified traits (such as head type, eye type, etc.) ${ }^{42}$ In other words, there are as many essential properties of a natural kind as there are ontologically distinct morphological structures which compose the members of that kind: typically, the higher-up a kind is in the evolutionary chain, the more essential properties it will possess. But secondly, and more importantly, given the nature of phenomodulatory dispositions, the essential properties of a natural kind must be those individually responsible for the morphological development of particular structural type of phenotypic feature - for these are properties which are "directed toward" determinable, not determinate end states.

Thus, the essence of a natural kind cannot be identified with a set of particularised morphological structures, but must instead be defined by a set of discrete morphogenetic developmental units, each individually responsible for the potential production of a unified gradient of an interrelated set of quantitative and qualitative permutations on a general architectural theme. In other words, each property that is "of the essence" of a natural kind establishes a wide-ranging and complex structural morphospace with respect to the specified development of each of the morphological features within the compositional make-up of the members of that kind. Utilising the conceptual framework of dynamic systems theory, where morphospace modelling of developmental modules is becoming increasingly important, this claim can be made more precise ${ }^{43}$ : the essential properties of natural kinds are characterised by higher-order 'epigenetic landscapes' whose topological peaks and troughs represent the various developmental pathways towards the various possible particularised end-states of the morphological structures for which those properties are responsible.

Each essential property of a natural kind - that is, each of its phenomodulatory dispositions - is thus defined by a multi-faceted, determinable developmental landscape whose various 'attractor basins' represent the variety of determinate forms which those properties' morphological structures may assume (in correlation with distinct sets of developmental stimuli). The essence of a natural kind then functions as a type of dynamic bauplan which causally undergirds the "static" morphological features typically associated with a set, or sets of organisms: its role is to establish an organism-wide 'morphospace', shaping and constraining the various possible (and typical) courses of its structural development, and, in any particular

\footnotetext{
42 Accordingly, given our knowledge of the existence of highly conserved developmental mechanisms - such as "toolkit" genes, discussed earlier - every natural kind will share a significant proportion of their essential properties, these being ontological traces of their evolutionary origins.

${ }^{43}$ Davila-Velderrain et al. (2015), Huang (2012), Wang et al. (2011)
} 
instance, to be the prime mover in the environmentally-determined, particularised exploration of that space. ${ }^{44}$

Using this criteria, it may be that 'cell' marks out a biological natural kind, defined by a single essential property: we now have quantitative mappings of the various developmental potentialities of qualitative cell-fate, complete with probability assignments with respect to those fates according to the influence of environmental stimuli, lending empirical credence to the existence of a phenomodulatory dispositional property. ${ }^{45} \mathrm{On}$ a larger scale, the specified homologue variants of developmental modules underwritten by homeotic selector genetic regulatory networks may qualify as essential properties for the sets of organisms which possess them. For instance, the morphological structure of the elytra - the scleratised forewings of beetles - looks to be "of the essence" of the organisms which possess them: these modules, though ubiquitously grounded in the $U b x$-abdA regulatory network, provide the causal basis for a wide-reaching reaction norm specifying the development of varying degrees of qualitatively distinct elytra shapes, sizes, colours, etc. among a variety of distinct species according to their varying "upstream" signalling pathways. ${ }^{46}$ In this way, particular "bottleneck" developmental modules responsible for the formation of the basic set of morphological structures which compose an organism are plausibly instances of phenomodulatory dispositions: though stable with respect to their possession among a wide range of organisms and with respect to their developmental control of a particular morphological structure, they nonetheless provide and specify a wide range of regulatory-dependent inter-structural variability - the precise mapping of which is now being carefully elucidated with the aid of dynamic systems theory.

With all of this in mind, it is a clear consequence of this conception of $\mathbb{E}$ that natural kinds cannot be identified with species, but rather must be considered on analogy with the conceptual middle of the taxonomic tree, where the phenomenon of environmental exploitation still occurs in a way that creates seemingly fundamental divides further down - for no collection of particularised instances of a phenomodulatory disposition's manifestation-type could represent the ontological division that 'natural kind' carves. Or to put it another way, again within the framework of dynamic systems theory, because the essence of a natural kind is defined by discrete sets of determinable developmental landscapes, particular collections of determinate end-states within those topologies cannot possibly capture the richer, more expansive conceptual space carved-out by the definition of 'natural kind': no limited set of developmental trajectories can hope to reconstruct the geometry of an entire topology. In this way, 'natural kind' is a more inclusive concept than 'species': the exploration of "morphogenetic space" afforded by the nature of phenomodulatory dispositions outstrips the narrow confines of the more particularised, well-entrenched (read: canalised) developmental pathways which typify the members of particular species.

Importantly, with this novel interpretation of $\mathbb{E}$ comes a novel characterisation of what the Aristotelian 'natural state' is. Because the ontological nature of 'essence' consists in specified potentiality that is, dispositionality - the kind-defining 'natural state' of an organism just is a dynamically plastic, generalised morphological developmental template. In other words, what it is to be a particular natural kind is to be an instantiation of a particular set of specifically patterned developmentally branching morphospaces. That said, it's clear that although this form of $\mathbb{E}$ declares stability, not variability to be ontologically fundamental, there is nuanced sense in which variation is fundamental in a certain respect: though the 'natural state' must be stable with respect to its possession, it is undoubtedly dynamic with respect to its activity - for, as we have seen, the possibility of the causal production of specified

${ }^{44}$ For a defence of the 'prime mover' aspect of this claim, see Austin (2015a).

45 Verd et al. (2014), Bhattacharya et al. (2011)

46 Wagner (2014), Deutsch (2005) 
variability is an intrinsic feature of the properties which compose it. ${ }^{47}$ Hence, to return to the earlier objections, the defender of $Æ$ need not deny that variation is the "raw material of evolutionary change", or even that it represents a "fundamental reality of nature" - indeed, the viability of the theoretical framework of $\mathbb{E}$ requires that the latter be the case.

Consequently, on this conception of the Aristotelian natural state, not only is morphological diversity among members of the same kind expected, it is accounted for: it is "of the nature" of kind-defining essential properties to contain within them the potentiality for environmentally-dependent variation on their associated morphological structures and, due to the specific functional role that phenomodulatory dispositions play, no particular instance of such structure within a member of a natural kind can be produced independently of its intra- and inter-cellular environment. In other words, because the kinddefining natural state of an organism just is a set of functional correlations between various values of environmental factors and various morphological responses, phenotypic diversity among members of the same kind is simply a consequence of their fundamental ontology ${ }^{48}$ Understood in this way, Sober's (1980: 374-377) aforementioned objection now looks to rest upon the horns of a false dilemma: no particularised form of a kind's morphological traits is "more natural" than any other - as each of them are in fact grounded in the 'natural state' - and although the 'natural state' functions as an intrinsic developmental plan, no particular instance of a natural kind develops independently of the causal context of its environment.

Not only does this conception of the Aristotelian 'natural state' allow and account for this sort of morphological variation within a natural kind, but due to the nature of phenomodulatory dispositions as "higher-order", functional properties, it likewise allows and accounts for genotypic variation therein thus, it is an essentialism that eschews any crude form of genetic reductionism. ${ }^{49}$ For the Aristotelian natural state, on the conception offered here, cannot be grounded in any particular genetic configuration: while it's true that if any particular genetic architecture is going to realise a phenomodulatory disposition it must perform a certain developmentally central causal role, any such configuration (and elements thereof) that does perform that role is an instance of that dispositional property. As in developmental systems theory, because a particular epigenetic landscape which maps-out the developmental fate of a system is specified by a higher-order, topological structure, any set of mechanistic underpinnings which comprise a system which satisfy that structure's dynamics realises that landscape. ${ }^{50}$ In this way, the aforementioned eventual evolutionary "disassociation" of developmental modules from their underlying generative mechanisms - and therefore, the phenomenon of intra-kind genetic diversity - can be understood as an inevitable consequence of the very ontology of 'essence'.

If we take on board this novel conception of the Aristotelian 'natural state', it's clear that the various historically damaging critiques of that model presented at the outset of this paper now simply collectively miss their mark. And all of this goes to show that, in the context of the proper niche constructed from a dispositional characterisation of 'essence' and a corresponding reformulated understanding of the Aristotelian 'natural state' as a kind of metaphysically dynamic bauplan - , and having

\footnotetext{
${ }^{47}$ In fact, it's for this very reason that Wagner (2014: 20) refers to his modelling of developmental modules as a theory of 'variational structuralism'.

48 According to this conception of the Aristotelian 'natural state', if we want to carve the world into proper natural kinds, it won't be enough to group together organisms which share exact morphologies - rather, as evo-devo has taught us, we must look conceptually underneath those morphologies.

${ }^{49}$ If, as Rosenberg (2001) argues, the process of natural selection operates on function, and is rather "blind" to structure, we shouldn't expect the essence of a natural kind, being so central to the process of ontogenic development, to be necessarily tied-up to a particular material realisation base.

${ }^{50}$ Jaeger \& Monk (2014), Dupré (2013), Rosa \& Exteberria (2011), Gilbert \& Bolker (2001)
} 
been refined by the selective pressures of the conceptual challenges of contemporary biology, an evolved essentialism is at hand.

\section{Summing Up}

As both the experimental utility and explanatory scope of the theoretical framework of evo-devo have substantially increased over the years, there has been a corresponding, though admittedly small resurgence of $\mathbb{E}$ in the philosophy of biology, one to which this paper aims to contribute..$^{51} \mathrm{~A}$ central theme of this movement has been the claim that not only are essentialism and evolution not in conflict, but that the latter in some way requires the former. Though the theory of $\mathbb{E}$ presented here differs importantly in both method and detail from these other works, it may nonetheless function as an expression of that same motif, understood as a more empirically discerning and metaphysically precise property-based account of how the ontological commitments of the contemporary framework of evo-devo lay bare what that requirement consists in. To that end, I have argued that if we re-conceptualise $\mathbb{E}$ 's original ontology of capacities in the form of contemporary dispositional properties, and subsequently understand the Aristotelian 'natural state' within the explanatory framework of evo-devo, we are afforded an essentialism that is not only theoretically plausible, in virtue of it being immune to its most prominent objections, but also empirically plausible, in virtue of it being in no way in conflict with, and perhaps even functioning as the conceptual foundation of contemporary evolutionary theory.

That said, in offering a general metaphysical theory of what it is to be an Aristotelian natural kind, I have purposely remained silent on the details of the further, future project of offering a specific empirical theory of which particular collections of developmental modules qualify as Aristotelian natural kinds. The reason for this silence is simple: the sole aim of this paper has been to propose and elucidate the metaphysical structure of a novel, empirically informed $\mathbb{E}$. Although such a theory may provide the conceptual foundations of a focused empirical research programme, this is a project which should not, and indeed cannot be carried out from the armchair: the subsequent success or failure of the search for and classification of biological natural kinds according to the proposed ontological divisions of this form of $\mathbb{E}$ is a matter which must be decided by more than mere metaphysics. With that in mind, there remain undoubtedly important areas for further conceptual work - among them, providing a theory of the origination of novel natural kinds, and the process by which this might take place, as well as elucidating potential empirical methods for discerning distinct natural kinds, and their various accompanying epistemological difficulties. ${ }^{52}$ Although I think there's philosophically fruitful work to be done in both of these areas, I here leave them for another time, with the hope that this paper might function as the conceptual bauplan for their subsequent study.

\section{Acknowledgements}

I am grateful for the generous support of the Analysis Trust.

\footnotetext{
${ }^{51}$ Most notably by Walsh (2006) and Boulter (2012), and to a lesser degree Devitt (2008).

${ }^{52}$ In the context of evo-devo, these sorts of studies may already be taking place. With respect to (a), investigating the effect of regulatory novelties on homology-generating pathways via mutation or epigenetic marking may be a viable way of discerning the arrival of novel phenomodulatory dispositions: see Wagner (2014), and Webster \& Goodwin (2006). With respect to (b), the method of distinguishing two homologous modules in virtue of their nonoverlapping sets of morphospace 'character states' may constitute an empirical method of detecting the presence of distinct phenomodulatory dispositions: see Wagner's (2014) discussion of representing the 'variational modalities' of homologous structures.
} 
This is a pre-print version of Aristotelian Essentialism: Essence in the Age of Evolution, Synthese (forthcoming). The final publication will be available at Springer via http://dx.doi.org/10.1007/s11229-016-1066-4

\section{References}

Altenberg, L. (1995). Genome Growth and the Evolution of the Genotype-Phenotype Map. In W. Banzhaf, \& F. Eeckman (Eds.), Evolution and Biocomputation: Computational Models of Evolution (pp. 205-250). Berlin: Springer.

Amundson, R. (2005). The Changing Role of the Embryo in Evolutionary Thought: Roots of Evo-Devo. Cambridge: Cambridge University Press.

Ashery-Padan, R., \& Gruss, P. (2001). Pax6 Lights-Up the Way for Eye Development. Current Opinion in Cell Biology, 706-714.

Aubin-Horth, N., \& Renn, S. (2009). Genomic Reaction Norms: Using Integrative Biology to Understand Molecular Mechanisms of Phenotypic Plasticity. Molecular Ecology, 3763-3780.

Austin, C. J. (2015a). The Dispositional Genome: Primus Inter Pares. Biology and Philosophy, 227-246.

Austin, C. J. (2015b). The Truthmaking Argument Against Dispositionalism. Ratio, 271-285.

Balme, D. (1987). Teleology and Necessity. In A. Gotthelf, \& J. Lennox (Eds.), Philosophical Issues in Aristotle's Biology (pp. 275-285). Cambridge: Cambridge University Press.

Bhattacharya, S., Zhang, Q., \& Andersen, M. (2011). A Deterministic Map of Waddington's Epigenetic Landscape for Cell Fate Specification. BMC Systems Biology, 1-11.

Bolker, J. (2000). Modularity in Development and Why It Matters to Evo-Devo. Integrative \& Comparitive Biology, 770776.

Boulter, S. J. (2012). Can Evolutionary Biology Do Without Aristotelian Essentialism? Royal Institute of Philosophy Supplement, 83-103.

Boyd, R. (1999). Homeostasis, Species, and Higher Taxa. In R. Wilson (Ed.), Species: New Interdisciplinary Essays (pp. 141-186). Cambridge: The MIT Press.

Cross, T. (2005). What is a Disposition? Synthese, 321-341.

Davila-Velderrain, J., Martinez-Garcia, J. C., \& Alvarez-Buyila, E. R. (2015). Modeling the Epigenetic Attractors Landscape: Toward a Post-Genomic Mechanistic Understanding of Development. Frontiers in Genetics, doi: 10.3389/fgene.2015.00160.

Devitt, M. (2008). Resurrecting Biological Essentialism. Philosophy of Science, 344-382.

Deutsch, J. (2005). Hox and Wings. BioEssays, 673-675

Dupre, J. (2013). Living Causes. Proceedings of the Aristotelian Society Supplementary Volume, 19-38.

Eagle, A. (2009). Causal Structuralism, Dispositional Actualism, and Counterfactual Conditionals. In T. Hanfield (Ed.), Dispositions and Causes (pp. 65-99). Oxford: Oxford University Press.

Eble, G. (2005). Morphological Modularity and Macroevolution: Conceptual and Empirical Aspects. In W.

Callebaut, \& D. Rasskin-Gutman (Eds.), Modularity: Understanding the Development and Evolution of Natural Complex Systems (pp. 221-239). Cambridge: MIT Press.

Edelman, G., \& Gally, J. (2001). Degeneracy and Complexity in Biological Systems. Proceedings of the National Academy of the Sciences, 98(24), 13763-13768.

Elder, C. (2008). Biological Species are Natural Kinds. Southern Journal of Philosophy, 339-362.

Ellis, B. (2010). Causal Powers and Categorical Properties. In A. Marmodoro (Ed.), The Metaphysics of Powers: Their Grounding and Their Manifestations (pp. 133-142). New York: Routledge.

Fusco, G., \& Minelli, A. (2010). Phenotypic Plasticity in Development and Evolution: Facts and Concepts. Philosophical Transactions of the Royal Society B, 547-556.

Galis, F., \& Metz, J. (2001). Testing the Vulnerability of the Phylotypic Stage: On Modularity and Evolutionary Conservation. Journal of Experimental Zoology, 195-204.

Gilbert, S., \& Bolker, J. (2001). Homologies of Process and Modular Elements of Embryonic Construction. Journal of Experimental Zoology, 1-12.

Gould, S. J. (1985). The Flamingo's Smile: Reflections in Natural History. New York: W.W. Norton \& Co.

Griffiths, P. (2002). What is Innateness. Monist, 70-85.

Gurdon, J., \& Bourillot, P. (2001). Morphogen Gradient Interpretation. Nature, 413, 797-803.

Hacking, I. (2007). Natural Kinds: Rosy Dawn, Scholastic Twilight. Royal Institute of Philosophy Supplement, 203-239.

Halder, G., Callaerts, P., \& Gehring, W. (1995). Induction of Ectopic Eyes by Targeted Expression of the Eyeless Gene in Drosophilia. Science, 1788-1792.

Huang, S. (2012). The Molecular and Mathematical Basis of Waddington's Epigenetic Landscape: A Framework for Post-Darwinian Biology? Bioessays, 149-157. 
This is a pre-print version of Aristotelian Essentialism: Essence in the Age of Evolution, Synthese (forthcoming). The final publication will be available at Springer via http://dx.doi.org/10.1007/s11229-016-1066-4

Hull, D. (1999). On the Plurality of Species: Questioning the Party Line. In R. Wilson (Ed.), Species: New Interdisciplinary Essays (pp. 23-48). Cambridge: MIT Press.

Jacobs, J. (2011). Powerful Qualities, Not Pure Powers. The Monist, 81-102.

Jaeger, J., \& Monk, N. (2014). Bioattractors: Dynamical Systems Theory and the Evolution of Regulatory Processes. Journal of Physiology, 2267-2281.

Kalinka, A., Varga, K., Gerrard, D., Preibisch, S., Corcoran, D., Jarrells, J., et al. (2010). Gene Expression Divergence Recapituates the Developmental Hourglass Model. Nature, 811-814.

Lennox, J. (2001). Material and Formal Natures in Aristotle's de Partibus Animalium. In J. Lennox, Aristotle's Pbilosophy of Biology (pp. 182-204). Cambridge: Cambridge University Press.

Lennox, J. G. (1987). Kinds, Forms of Kinds, and the More and the Less in Aristotle's Biology. In A. Gotthelf, \& J. G. Lennox (Eds.), Philosophical Issues in Aristotle's Biology (pp. 339-359). Cambridge: Cambridge University Press.

Lewis, D. (2000). Causation as Influence. The Journal of Philosophy, 182-197.

Lewontin, R. (1978). Adaption. Scientific American, 212-228.

Manley, D., \& Wasserman, R. (2008). On Linking Dispositions and Conditionals. Mind, 59-84.

Mann, R., \& Carroll, B. (2002). Molecular Mechanics of Selector Gene Function and Evolution. Current Opinion in Genetics \& Development, 592-600.

Martin, C. (2008). The Mind in Nature. Oxford: Oxford University Press.

Mayr, E. (1976). Evolution and the Diversity of Life. Cambridge: Harvard University Press.

Mayr, E. (1992). The Idea of Teleology. Journal of the History of Ideas, 117-135.

McGhee, G. (2006). The Geometry of Evolution: Adaptive Landscapes and Theoretical Morphospaces. Cambridge: Cambridge University Press.

Müller, G. (2003). Homology: The Evolution of Morphological Organization. In G. B. Muller, \& S. A. Newman (Eds.), Origination of Organismal Form: Beyond the Gene in Developmental and Evolutionary Biology (pp. 51-69). Cambridge: MIT Press.

Müller, G. (2008). Evo-Devo as a Discipline. In A. Minelli, \& G. Fusco (Eds.), Evolving Pathways: Key Themes in Evolutionary Developmental Biology (pp. 3-29). Cambridge: Cambridge University Press.

Müller, G., \& Newman, S. A. (1999). Generation, Integration, Autonomy: Three Steps in the Evolution of Homology. Novartis Foundation Symposia, 65-73.

Mumford, S., \& Anjum, R. (2011). Getting Causes From Powers. Oxford: Oxford University Press.

Nagel, E. (1977). Goal-Directed Processes in Biology. The Journal of Philosophy, 261-279.

Newman, S., \& Muller, G. (2006). Genes and Form: Inherency in the Evolution of Developmental Mechanisms. In E. Neumann-Held, \& C. Rehmann-Sutter (Eds.), Genes in Development: Re-Reading the Molecular Paradigm (pp. 38-77). Durham: Duke University Press.

Newman, S., Forgacs, G., \& Muller, G. (2006). Before Programs: The Physical Origination of Multicellular Forms. International Journal of Developmental Biology, 289-299.

Oderberg, D. (2009). The Non-Identity of the Categorical and Dispositional. Analysis, 677-684.

Okasha, S. (2002). Darwinian Metaphysics: Species and the Question of Essentialism. Sythese, 191-213.

Pellegrin, P. (1987). Logical Difference and Biological Difference: the Unity of Aristotle's Thought. In A. Gotthelf, \& J. Lennox (Eds.), Philosophical Issues in Aristotle's Biology (pp. 313-338). Cambridge: Cambridge University Press.

Pigliucci, M. (2001). Phenotypic Plasticity: Beyond Nature and Nurture. Baltimore: Johns Hopkins University Press.

Raff, R., \& Sly, B. (2000). Modularity and Dissociation in the Evolution of Gene Expression Territories in Development. Evolution and Development, 102-113.

Rasskin-Gutman, D. (2003). Boundary Constraints for the Emergence of Form. In G. Muller, \& S. Newman (Eds.), The Origination of Organismal Form (pp. 305-322). Cambridge: MIT Press.

Rasskin-Gutman, D. (2005). Modularity: Jumping Forms within Morphospace. In W. Callebaut, D. Rasskin-

Gutman, \& H. Simon (Eds.), Modularity: Understanding the Development and Evolution of Natural Complex Systems (pp. 207-219). Cambridge: MIT Press.

Rosa, L., \& Etxeberria, A. (2011). Pattern and Process in Evo-Devo: Descriptions and Explanations. In H. de Regt, S. Hartmann, \& S. Okasha (Eds.), EPS A Pbilosophy of Science: Amsterdam 2009 (pp. 263-274)

Rosenberg, A. (2001). On Multiple Realization and the Special Sciences. The Journal of Pbilosophy, 365-373. 
This is a pre-print version of Aristotelian Essentialism: Essence in the Age of Evolution, Synthese (forthcoming). The final publication will be available at Springer via http://dx.doi.org/10.1007/s11229-016-1066-4

Salazar-Ciudad, I., \& Jernvall, J. (2013). The Causality Horizon and the Developmental Bases of Morphological Evolution. Biological Theory, 286-292.

Schlichting, C., \& Smith, H. (2002). Phenotypic Plasticity: Linking Molecular Mechanisms with Evolutionary Outcomes. Evolutionary Ecology, 189-211.

Schrenk, M. (2010). The Powerlessness of Necessity. Nous, 725-739.

Shubin, N., Tabin, C., \& Carroll, S. (2009). Deep Homology and the Origins of Evolutionary Novelty. Nature, 818823.

Sober, E. (1980). Evolution, Population Thinking, and Essentialism. (E. Sober, Ed.) Philosophy of Science, 350-383.

Striedter, G. (1998). Stepping into the Same River Twice: Homologues as Recurring Attractors in Epigenetic Landscapes. Brain, Behavior and Evolution, 218-231.

Tabata, T. (2001). Genetics of Morphogen Gradients. Nature, 620-630.

Vetter, B. (2013). Multi-Track Dispositions. The Pbilosophical Quarterly, 330-352.

Verd, B., Crombach, A., \& Jaeger, J. (2014). Classification of Transient Behaviours in a Time-Dependent Toggle Switch Model. BMC Systems Biology, 1-19.

Von Dassow, G., \& Munro, E. (1999). Modularity in Animal Development and Evolution: Elements of a Conceptual Framework for Evo Devo. Journal of Experimental Zoology, 307-325.

von Dassow, G., Meir, E., Munro, E. M., \& Odell, G. M. (2000). The Segment Polarity Network is a Robust Developmental Module. Nature, 188-192.

Wagner, G. (2000). Characters, Units and Natural Kinds: An Introduction. In G. Wagner (Ed.), The Character Concept in Evolutionary Biology (pp. 1-10). Connecticut: Academic Press.

Wagner, G. (2007). The Developmental Genetics of Homology. Nature Review of Genetics, 473-479.

Wagner, G. (2014). Homology, Genes, and Evolutionary Innovation. Princeton: Princeton University Press.

Wagner, G., \& Altenberg, L. (1996). Complex Adaptations and the Evolution of Evolvability. Evolution, 967-976.

Walsh, D. (2006). Evolutionary Essentialism. British Journal of the Philosophy of Science, 425-448.

Walsh, D. (2012). Mechanism and Purpose: A Case of Natural Teleology. Studies in History and Philosophy of Biological Biomedical Sciences, 173-181.

Wang, J., Zhang, K., Xu, L., \& Wang, E. (2011). Quantifying the Waddington Landscape and Biological Paths for Development and Differentiation. Proceedings of the National Academy of Sciences of the United States of America, 8257-8262.

Webster, G., \& Goodwin, C. (2006). The Origin of Species: A Structuralist Approach. In E. Neumann-Held, \& C. Rehmann-Sutter (Eds.), Genes in Development: Re-Reading the Molecular Paradigm (pp. 99-134). Durham: Duke University Press.

West-Eberhard, M. (2003). Developmental Plasticity and Evolution. New York: Oxford University Press.

Whitacre, J., \& Bender, A. (2010). Networked Buffering: A Basic Mechanism for Distributed Robustness in Complex Adaptive Systems. Theoretical Biology and Medical Modelling, 1-20.

Whitman, D. W., \& Agrawal, A. A. (2009). What is Phenotypic Plasiticty and Why Is It Important? In T. N. Ananthakrishna, \& D. W. Whitman (Eds.), Phenotypic Plasticity of Insects: Mechanisms and Consequences (pp. 163). Enfield: Science Publishers.

Wilkins, J. (2013). Biological Essentialism and the Tidal Change of Natural Kinds. Science \& Education, 221-240.

Wilson, R. (1999). Realism, Essence, and Kind: Resucitating Species Essentialism? In R. Wilson (Ed.), Species: New Interdisciplinary Essays (pp. 187-208). Cambridge: MIT Press.

Wilson, R., Barker, M., \& Brigandt, I. (2007). When Traditional Essentialism Fails: Biological Natural Kinds. Philosophical Topics, 189-215.

Winther, R. G. (2001). Varities of Modules: Kinds, Levels, Origins, and Behaviors. Journal of Experimental Zoology, 116-129.

Woodward, J. (2003). Making Things Happen: A Theory of Causal Explanation. Oxford: Oxford University Press.

Woodward, J. (2010). Causation in Biology: Stability, Specificity, and the Choice of Levels of Explanation. Biology and Philosophy, 287-318. 